\title{
Expressing the operations of quantum computing in multiparticle geometric algebra
}

\author{
Shyamal S. Somaroo \\ $B C M P$, Harvard Medical School \\ 240 Longwood Ave., Boston, MA 02115 \\ David G. Cory \\ Dept. of Nuclear Engineering \\ Massachusetts Institute of Technology \\ Cambrdige, MA 02139 \\ Timothy F. Havel* \\ BCMP, Harvard Medical School \\ 240 Longwood Ave., Boston, MA 02115
}

(September 7, 2018)

\begin{abstract}
We show how the basic operations of quantum computing can be expressed and manipulated in a clear and concise fashion using a multiparticle version of geometric (aka Clifford) algebra. This algebra encompasses the product operator formalism of NMR spectroscopy, and hence its notation leads directly to implementations of these operations via NMR pulse sequences.
\end{abstract}

02.40.Dr,03.65.Fd,31.15.-p,33.25.+k

Typeset using REVTEX

*To whom correspondence should be addressed at havel@menelaus .med . harvard.edu (617/4323242 office, 617/738-0516 FAX). 
Geometric (aka Clifford) algebra is a generalization of vector algebra to arbitrary dimensions and signatures, which provides a concise and geometrically transparent notation for describing a wide range of physical phenomena (for introductions and examples, see [1 [6]). Multiparticle geometric algebra is a recent further generalization that enables one to deal with interacting two state quantum systems [7,8]. Since most models of a quantum computer are based on such systems [9 [1], it is of interest to formulate the basic operations of quantum computing in these terms, with the goal of gaining deeper insight into them. Given the recently discovered methods of emulating a quantum computer via NMR spectroscopy [12 [14, it is further of interest to note that one of the main tools that NMR spectroscopists have developed to aid them in understanding their experiments, known as the product operator formalism [15 [18], is a subalgebra of a multiparticle geometric algebra. Geometric algebra also encompasses the quaternion methods often used by NMR spectroscopists to calculate the effects of composite pulses on product operators [19 21]. Thus, as illustrated in this letter, it is generally straightforward to translate a quantum logic operation expressed in the multiparticle geometric algebra into an NMR implementation thereof.

We shall begin with a physically motivated definition of the geometric algebra $\mathcal{G}(3)$ of a single particle. This algebra is isomorphic to the Pauli matrix algebra, where the isomorphism is obtained by identifying the Pauli matrices $\underline{\boldsymbol{\sigma}}_{1}, \underline{\boldsymbol{\sigma}}_{2}, \underline{\boldsymbol{\sigma}}_{3}$ with an orthonormal basis $\boldsymbol{\sigma}_{1}, \boldsymbol{\sigma}_{2}, \boldsymbol{\sigma}_{3}$ of a Euclidean vector space. Note that the product of all three Pauli matrices $\underline{\boldsymbol{\sigma}}_{1} \underline{\boldsymbol{\sigma}}_{2} \underline{\boldsymbol{\sigma}}_{3}$ is just the imaginary unit " $\imath$ " times the identity matrix, which we denote by $\underline{\boldsymbol{\iota}} \equiv \boldsymbol{\imath} \underline{\mathbf{1}}$. This enables us to further identify the corresponding element $\boldsymbol{\iota} \equiv \boldsymbol{\sigma}_{1} \boldsymbol{\sigma}_{2} \boldsymbol{\sigma}_{3}$ of the geometric algebra $\mathcal{G}(3)$, which is commonly called the unit pseudo-scalar, with the imaginary unit itself. In this way $\mathcal{G}(3)$ becomes an algebra over the real numbers, even though the Pauli matrix algebra is usually regarded as a complex algebra.

The (nonrelativistic) $N$-particle geometric algebra $\mathcal{G}^{N}(3)$ consists of a direct product of $N$ copies of $\mathcal{G}(3):$ :

$$
\mathcal{G}^{N}(3) \equiv \mathcal{G}(3) \otimes \cdots \otimes \mathcal{G}(3) \quad(N \text { factors })
$$

Throughout this letter, we shall assume that $1 \leq i, j, k \leq 3,1 \leq \ell, m, n \leq N$, and that $\alpha, \beta, \ldots$ are real scalars. Then the multiparticle geometric algebra can also be defined by the following five straightforward rules:

$$
\begin{aligned}
& \left\{\boldsymbol{\sigma}_{i}^{\ell}\right\} \text { is a basis for a real vector space; } \\
& \boldsymbol{\sigma}_{i}^{\ell}\left(\boldsymbol{\sigma}_{j}^{m} \boldsymbol{\sigma}_{k}^{n}\right)=\left(\boldsymbol{\sigma}_{i}^{\ell} \boldsymbol{\sigma}_{j}^{m}\right) \boldsymbol{\sigma}_{k}^{n} ; \\
& \boldsymbol{\sigma}_{i}^{\ell}\left(\alpha \boldsymbol{\sigma}_{j}^{m}+\beta \boldsymbol{\sigma}_{k}^{n}\right)=\alpha \boldsymbol{\sigma}_{i}^{\ell} \boldsymbol{\sigma}_{j}^{m}+\beta \boldsymbol{\sigma}_{i}^{\ell} \boldsymbol{\sigma}_{k}^{n} \text { and } \\
& \left(\alpha \boldsymbol{\sigma}_{i}^{\ell}+\beta \boldsymbol{\sigma}_{j}^{m}\right) \boldsymbol{\sigma}_{k}^{n}=\alpha \boldsymbol{\sigma}_{i}^{\ell} \boldsymbol{\sigma}_{k}^{n}+\beta \boldsymbol{\sigma}_{j}^{m} \boldsymbol{\sigma}_{k}^{n} ; \\
& \boldsymbol{\sigma}_{i}^{\ell} \boldsymbol{\sigma}_{j}^{\ell}+\boldsymbol{\sigma}_{j}^{\ell} \boldsymbol{\sigma}_{i}^{\ell}=2 \delta_{i j} \quad(\text { the Kronecker delta }) ; \\
& \text { for all } \ell \neq m: \quad \boldsymbol{\sigma}_{i}^{\ell} \boldsymbol{\sigma}_{j}^{m}-\boldsymbol{\sigma}_{j}^{m} \boldsymbol{\sigma}_{i}^{\ell}=0 .
\end{aligned}
$$

The NMR product operator formalism relies upon a large repertoire of relatively complicated rules for predicting the evolution of the spins, which vary with the interaction (e.g. strong

\footnotetext{
1 This definition can be derived from a relativistic multiparticle theory based on the geometric algebra of $N$ copies of space-time [7],8].
} 
or weak coupling), the total angular momentum (for spin $>\frac{1}{2}$ ), and so on. With a little practice, however, all the rules of the product operator formalism can be readily derived as they are needed from the five rules given in Eqs. (2- 6).

By our definition of $\mathcal{G}(3)$ above, a faithful matrix representation of this $\left(2^{3}\right)$-dimensional real algebra is obtained by mapping the basis vectors to the Pauli matrices: $\boldsymbol{\sigma}_{k} \rightarrow \underline{\boldsymbol{\sigma}}_{k}$. A matrix representation of $\mathcal{G}^{N}(3)$ is obtained simply by taking $N$-fold Kronecker products of these matrices. Since the representation by Pauli matrices is a complex algebra, however, its real dimension is $2 \cdot 4^{N}=2^{2 N+1}$, which for $N>1$ is less than the dimension $2^{3 N}$ of $\mathcal{G}^{N}(3)$. It follows that the direct product representation is no longer faithful. The extra degrees of freedom are due to the fact that, unlike the matrix representation, there is a different complex unit $\iota^{\ell} \equiv \boldsymbol{\sigma}_{1}^{\ell} \boldsymbol{\sigma}_{2}^{\ell} \boldsymbol{\sigma}_{3}^{\ell}$ for every particle. Since the matrix algebra is believed to contain all possible quantum states, operators and propagators, these degrees of freedom have no known physical relevance. They can easily be removed by multiplying through by a primitive idempotent of the form

$$
\mathbf{C} \equiv \frac{1}{2}\left(1-\iota^{1} \iota^{2}\right) \frac{1}{2}\left(1-\iota^{1} \iota^{3}\right) \cdots \frac{1}{2}\left(1-\iota^{1} \iota^{N}\right)
$$

This correlator commutes with everything in the product algebra, and projects it onto an ideal of the correct dimension. The projection can be interpreted physically as locking the phases of the various particles together. For this reason, we shall not specify which factor our imaginary unit $\iota$ comes from, since all choices are rendered equal by the correlator whose presence in all our expressions will also not, in the interests of brevity, be written out explicitly. Further discussion of these issues may be found in [0,8].

Another class of primitive idempotents that we shall need are

$$
\mathbf{E}_{ \pm}^{m} \equiv \frac{1}{2}\left(1 \pm \boldsymbol{\sigma}_{3}^{m}\right)
$$

and products thereof from different factors. These are easily shown to have the following properties:

$$
\left(\mathbf{E}_{ \pm}^{m}\right)^{2}=\mathbf{E}_{ \pm}^{m}, \quad \mathbf{E}_{ \pm}^{m} \mathbf{E}_{\mp}^{m}=0, \quad \mathbf{E}_{+}^{m}+\mathbf{E}_{-}^{m}=1, \quad \boldsymbol{\sigma}_{3}^{m} \mathbf{E}_{ \pm}^{m}= \pm \mathbf{E}_{ \pm}^{m}
$$

A product of $N$ such idempotents, one for each particle, has the $\left(2^{N}\right) \times\left(2^{N}\right)$ matrix representation

$$
\underline{\mathbf{E}}_{\epsilon_{1}} \otimes \cdots \otimes \underline{\mathbf{E}}_{\epsilon_{N}} \leftrightarrow\left|\epsilon_{1} \cdots \epsilon_{N}\right\rangle\left\langle\epsilon_{1} \cdots \epsilon_{N}\right|
$$

where $\epsilon_{m}= \pm 1$ and $\underline{\mathbf{E}}_{ \pm} \equiv \frac{1}{2}\left(\underline{\mathbf{1}} \pm \underline{\boldsymbol{\sigma}}_{3}\right)$. To illustrate the utility of these idempotents, we will begin by using them to parametrize an arbitrary entity from the multiparticle geometric algebra $\mathcal{G}^{N}(3)$ in terms of "rotors" from its even subalgebra [2]. We shall do this for only two particles, from which the general case should be clear.

Given an arbitrary multivector $\mathbf{M} \in \mathcal{G}(3) \otimes \mathcal{G}(3)$, we may write

$$
\mathbf{M}=\mathbf{M}\left(\mathbf{E}_{+}^{1}+\mathbf{E}_{-}^{1}\right)\left(\mathbf{E}_{+}^{2}+\mathbf{E}_{-}^{2}\right)=\mathbf{M E}_{+}^{1} \mathbf{E}_{+}^{2}+\cdots+\mathbf{M E}_{-}^{1} \mathbf{E}_{-}^{2} .
$$

Let $\langle\mathbf{M}\rangle_{+} \equiv \frac{1}{2}(\mathbf{M}+\hat{\mathbf{M}})$ denote the projection of $\mathbf{M}$ onto the even subalgebra $(\mathcal{G}(3) \otimes \mathcal{G}(3))^{+}=$ 
$\mathcal{G}^{+}(3) \otimes \mathcal{G}^{+}(3)$, where $\hat{\mathbf{M}}$ is the grade involution of $\mathbf{M}$. Then, using the fact that $\mathbf{E}_{ \pm}^{m} \mathbf{E}_{\mp}^{m}=0$ and $\hat{\mathbf{E}}_{ \pm}^{m}=\mathbf{E}_{\mp}^{m}$, each term above may be written as

$$
\begin{aligned}
\mathbf{M E}_{\epsilon_{1}}^{1} \mathbf{E}_{\epsilon_{2}}^{2} & =\left(\mathbf{M} \mathbf{E}_{\epsilon_{1}}^{1} \mathbf{E}_{\epsilon_{2}}^{2}\right)\left(\mathbf{E}_{\epsilon_{1}}^{1} \mathbf{E}_{\epsilon_{2}}^{2}\right) \\
& =\left(\mathbf{M} \mathbf{E}_{\epsilon_{1}}^{1} \mathbf{E}_{\epsilon_{2}}^{2}+\mathbf{M E}_{-\epsilon_{1}}^{1} \mathbf{E}_{-\epsilon_{2}}^{2}\right)\left(\mathbf{E}_{\epsilon_{1}}^{1} \mathbf{E}_{\epsilon_{2}}^{2}\right) \\
& =2\left\langle\mathbf{M} \mathbf{E}_{\epsilon_{1}}^{1} \mathbf{E}_{\epsilon_{2}}^{2}\right\rangle_{+}\left(\mathbf{E}_{\epsilon_{1}}^{1} \mathbf{E}_{\epsilon_{2}}^{2}\right)
\end{aligned}
$$

$\left(\epsilon_{1}, \epsilon_{2} \in\{ \pm 1\}\right)$. It follows that

$$
\mathbf{M}=\left(\boldsymbol{\psi}_{++} \mathbf{E}_{+}^{1} \mathbf{E}_{+}^{2}+\boldsymbol{\psi}_{+-} \mathbf{E}_{+}^{1} \mathbf{E}_{-}^{2}+\boldsymbol{\psi}_{-+} \mathbf{E}_{-}^{1} \mathbf{E}_{+}^{2}+\boldsymbol{\psi}_{--} \mathbf{E}_{-}^{1} \mathbf{E}_{-}^{2}\right)
$$

where each rotor $\boldsymbol{\psi}_{\epsilon_{1} \epsilon_{2}}$ is in $\mathcal{G}^{+}(3) \otimes \mathcal{G}^{+}(3)$, and each $\boldsymbol{\psi}_{\epsilon_{1} \epsilon_{2}} \mathbf{E}_{\epsilon_{1}}^{1} \mathbf{E}_{\epsilon_{2}}^{2}$ can be viewed as a twoparticle spinor [7,8]. A similar expansion holds for any number of particles and, when translated into matrices via Eq. (10) above, corresponds to an expansion of the matrix into a sum of matrices each of which contains one column from the original matrix, and is otherwise zero.

If we multiply $\mathbf{M}$ by its reverse $\tilde{\mathbf{M}}$, we obtain

$$
\begin{aligned}
\mathbf{M} \tilde{M} & =\left(\boldsymbol{\psi}_{++} \mathbf{E}_{+}^{1} \mathbf{E}_{+}^{2}+\cdots+\boldsymbol{\psi}_{--} \mathbf{E}_{-}^{1} \mathbf{E}_{-}^{2}\right)\left(\mathbf{E}_{+}^{1} \mathbf{E}_{+}^{2} \tilde{\boldsymbol{\psi}}_{++}+\cdots+\mathbf{E}_{-}^{1} \mathbf{E}_{-}^{2} \tilde{\boldsymbol{\psi}}_{--}\right) \\
& =\boldsymbol{\psi}_{++} \mathbf{E}_{+}^{1} \mathbf{E}_{+}^{2} \tilde{\boldsymbol{\psi}}_{++}+\boldsymbol{\psi}_{+-} \mathbf{E}_{+}^{1} \mathbf{E}_{-}^{2} \tilde{\boldsymbol{\psi}}_{+-}+\boldsymbol{\psi}_{-+} \mathbf{E}_{-}^{1} \mathbf{E}_{+}^{2} \tilde{\boldsymbol{\psi}}_{-+}+\boldsymbol{\psi}_{--} \mathbf{E}_{-}^{1} \mathbf{E}_{-}^{2} \tilde{\boldsymbol{\psi}}_{--}
\end{aligned}
$$

Since the reverse corresponds to the Hermitian conjugate of matrices, this expression represents a general Hermitian matrix. A rank one Hermitian matrix is obtained from any single term of the expression, e.g. $\boldsymbol{\psi}_{++} \mathbf{E}_{+}^{1} \mathbf{E}_{+}^{2} \tilde{\boldsymbol{\psi}}_{++}$. Provided that $\boldsymbol{\psi}_{++} \tilde{\boldsymbol{\psi}}_{++}=1$, the scalar part of this expression is $\frac{1}{4}$, which corresponds to a matrix trace of one. Thus this expression represents the density matrix of a pure state, while a general density matrix is a convex combination of terms as in Eq. (14) above.

A simple rotation by an angle $\alpha$ about an axis $\mathbf{a}=\Sigma_{i} a_{i} \boldsymbol{\sigma}_{i}(\|\mathbf{a}\|=1)$ in a one-particle space is given by the exponential of its generator, e.g. $\exp (-\alpha \boldsymbol{\iota} \mathbf{a} / 2)$, just as is commonly done with Pauli matrices. Due to the commutivity of vectors $\boldsymbol{\sigma}_{i}^{m}, \boldsymbol{\sigma}_{j}^{n}$ from different particle spaces, one cannot express arbitrary rotations between particle spaces in the algebra. An exception to this rule are the particle interchange operators, which in the case of two particles is given by

$$
\boldsymbol{\Pi}^{1,2} \equiv \frac{1}{2}\left(1+\boldsymbol{\sigma}_{1}^{1} \boldsymbol{\sigma}_{1}^{2}+\boldsymbol{\sigma}_{2}^{1} \boldsymbol{\sigma}_{2}^{2}+\boldsymbol{\sigma}_{3}^{1} \boldsymbol{\sigma}_{3}^{2}\right)
$$

An important variation on this is given by the scalar coupling Hamiltonian in NMR spectroscopy, $\mathbf{J}^{1,2} \equiv \pi J^{1,2}\left(2 \boldsymbol{\Pi}^{1,2}-1\right) / 2$, where $J^{1,2}$ is a coupling constant in Hertz [17]. Since it is easily shown that $\Pi^{1,2}$ squares to one, the corresponding time-dependent propagator is

$$
\exp \left(\boldsymbol{\iota} \mathbf{J}^{1,2} t\right)=e^{-\iota \pi J^{1,2} t / 2}\left(\cos \left(\pi J^{1,2} t\right)+\iota \Pi^{1,2} \sin \left(\pi J^{1,2} t\right)\right) .
$$

${ }^{2}$ For definitions of the grade involution, the reverse, and other common geometric algebra terms, see [4]. The identity $(\mathcal{G}(3) \otimes \mathcal{G}(3))^{+}=\mathcal{G}^{+}(3) \otimes \mathcal{G}^{+}(3)$ holds because for all $1 \leq i, j \leq 3$ and $1 \leq m, n \leq N, \boldsymbol{\sigma}_{i}^{m} \boldsymbol{\sigma}_{j}^{n} \mathbf{C}=-\iota^{m} \boldsymbol{\sigma}_{i}^{m} \iota^{n} \boldsymbol{\sigma}_{j}^{n} \mathbf{C}$ where $\mathbf{C}$ is the correlator defined in Eq. (7). 
In the product operator formalism, one generally assumes weak coupling, i.e. $\left|\omega^{1}-\omega^{2}\right| \gg$ $\pi J^{1,2}$, where $\omega^{1}, \omega^{2}$ are the resonance frequencies of the spins. This enables the usual two-spin Hamiltonian of NMR spectroscopy, i.e. $\mathbf{H}^{1,2} \equiv \mathbf{J}^{1,2}+\mathbf{K}^{1,2}$ where $\mathbf{K}^{1,2} \equiv \frac{1}{2}\left(\omega^{1} \boldsymbol{\sigma}_{3}^{1}+\omega^{2} \boldsymbol{\sigma}_{3}^{2}\right)$ denotes the Zeeman Hamiltonian, to be replaced by its first-order approximation

$$
\mathbf{H}_{\text {weak }}^{1,2} \equiv \mathbf{K}^{1,2}+\frac{1}{2} \pi J^{1,2} \boldsymbol{\sigma}_{3}^{1} \boldsymbol{\sigma}_{3}^{2}
$$

Since this approximation is diagonal in the usual $\boldsymbol{\sigma}_{3}^{1}+\boldsymbol{\sigma}_{3}^{2}$ basis, its propagator can be written down in closed form (see e.g. [15 [17). Geometric algebra is however not limited to weak coupling, although strong coupling of course complicates the propagators substantially. This will be demonstrated shortly, when we show how to diagonalize a general two-spin Hamiltonian.

We next show how to implement a rotation in one particle space conditional on the state of another it is correlated with. The simplest example is the controlled-NOT (or XOR) quantum gate, which is generated by a transition Hamiltonian of the form

$$
\mathbf{H}_{\mathrm{tr}}^{1 \mid 2} \equiv \boldsymbol{\sigma}_{1}^{1} \mathbf{E}_{-}^{2}=\frac{1}{2} \boldsymbol{\sigma}_{1}^{1}\left(1-\boldsymbol{\sigma}_{3}^{2}\right)
$$

Since $\left(\boldsymbol{\sigma}_{1}^{1}\right)^{2}=1$ and $\boldsymbol{\sigma}_{1}^{1}$ commutes with the idempotent $\mathbf{E}_{-}^{2}$, the corresponding propagator is given by

$$
\mathbf{R}_{1}^{1 \mid 2}(\alpha)=e^{-\iota \alpha \mathbf{H}_{\mathrm{tr}}^{1 \mid 2} / 2}=e^{-\iota \alpha \boldsymbol{\sigma}_{1}^{1} / 2} \mathbf{E}_{-}^{2}+\mathbf{E}_{+}^{2}
$$

This rotates the first spin about $\boldsymbol{\sigma}_{1}^{1}$ in those states in which the second spin is along the $-\boldsymbol{\sigma}_{2}^{3}$ axis. The controlled-NOT $\mathbf{R}_{1}^{1 \mid 2} \equiv \mathbf{R}_{1}^{1 \mid 2}(\pi)$ is obtained when $\alpha=\pi$, in which case the density matrix of the basis states $\mathbf{E}_{\epsilon_{1}}^{1} \mathbf{E}_{\epsilon_{2}}^{2}$ are transformed as follows:

$$
\mathbf{R}_{1}^{1 \mid 2}\left(\mathbf{E}_{\epsilon_{1}}^{1} \mathbf{E}_{\epsilon_{2}}^{2}\right) \tilde{\mathbf{R}}_{1}^{1 \mid 2}=\mathbf{E}_{\epsilon_{1} \epsilon_{2}}^{1} \mathbf{E}_{\epsilon_{2}}^{2}= \begin{cases}\mathbf{E}_{-\epsilon_{1}}^{1} \mathbf{E}_{\epsilon_{2}}^{2} & \text { if } \epsilon_{2}=-1 \\ \mathbf{E}_{+\epsilon_{1}}^{1} \mathbf{E}_{\epsilon_{2}}^{2} & \text { if } \epsilon_{2}=+1\end{cases}
$$

In order to obtain a controlled-NOT that preserves the phases of the basis states (as is usually assumed), one need only multiply $\mathbf{R}_{1}^{1 \mid 2}$ by the conditional phase $\operatorname{shift} \exp \left(\boldsymbol{\iota} \pi \mathbf{E}_{-}^{2} / 2\right)=$ $\iota \mathbf{E}_{-}^{2}+\mathbf{E}_{+}^{2}$. Similarly, the propagator for the controlled-controlled-NOT (or Toffoli gate) can be written as

$$
e^{\iota \pi\left(1-\boldsymbol{\sigma}_{1}^{1}\right) \mathbf{E}_{-}^{2} \mathbf{E}_{-}^{3} / 2}=\boldsymbol{\sigma}_{1}^{1} \mathbf{E}_{-}^{2} \mathbf{E}_{-}^{3}+\left(1-\mathbf{E}_{-}^{2} \mathbf{E}_{-}^{3}\right)
$$

while the Fredkin gate is given by the conditional particle interchange

$$
e^{\ell \pi\left(1-\Pi^{1,2}\right) \mathbf{E}_{+}^{3} / 2}=\Pi^{1,2} \mathbf{E}_{+}^{3}+\mathbf{E}_{-}^{3} .
$$

In [14], implementations of the controlled-NOT and Toffoli gates by NMR pulse sequences were given, which can also be easily validated by geometric algebra methods. In the case of the controlled-NOT, the implementation consists of a product of three propagators, which may be simplified as 


$$
\begin{aligned}
& \mathbf{S}_{1}^{1 \mid 2} \equiv e^{-\iota \pi \sigma_{1}^{1} / 4} e^{-\iota \pi \sigma_{3}^{1} \sigma_{3}^{2} / 4} e^{-\iota \pi \sigma_{2}^{1} / 4} \\
& =e^{-\iota \pi \sigma_{1}^{1} / 4}\left(e^{-\iota \pi \sigma_{3}^{1} \sigma_{3}^{2} / 4} e^{-\iota \pi \sigma_{2}^{1} / 4} e^{\iota \pi \sigma_{3}^{1} \sigma_{3}^{2} / 4}\right) e^{-\iota \pi \sigma_{3}^{1} \sigma_{3}^{2} / 4} \\
& =e^{-\imath \pi \sigma_{1}^{1} / 4} e^{\iota \pi \sigma_{1}^{1} \sigma_{3}^{2} / 4} e^{-\imath \pi \sigma_{3}^{1} \sigma_{3}^{2} / 4}\left(\mathbf{E}_{+}^{2}+\mathbf{E}_{-}^{2}\right) \\
& =\left(e^{-\boldsymbol{\iota} \pi \boldsymbol{\sigma}_{1}^{1} / 2} \mathbf{E}_{-}^{2}+\mathbf{E}_{+}^{2}\right)\left(e^{-\boldsymbol{\iota} \pi \boldsymbol{\sigma}_{3}^{1} / 4} \mathbf{E}_{+}^{2}+e^{\boldsymbol{\iota} \pi \boldsymbol{\sigma}_{3}^{1} / 4} \mathbf{E}_{-}^{2}\right) \\
& =e^{-\iota \pi \boldsymbol{\sigma}_{1}^{1} / 2} e^{\iota \pi \boldsymbol{\sigma}_{3}^{1} / 4} \mathbf{E}_{-}^{2}+e^{-\iota \pi \boldsymbol{\sigma}_{3}^{1} / 4} \mathbf{E}_{+}^{2} \text {, }
\end{aligned}
$$

where we have used Eq. (9) to get the relation $\exp \left(-\boldsymbol{\iota} \pi \boldsymbol{\sigma}_{3}^{1} \boldsymbol{\sigma}_{3}^{2} / 4\right) \mathbf{E}_{ \pm}^{2}=\exp \left(\mp \boldsymbol{\iota} \pi \boldsymbol{\sigma}_{3}^{1} / 4\right) \mathbf{E}_{ \pm}^{2}$. To get a controlled-NOT that preserves phases, we may left-multiply $\mathbf{S}_{1}^{1 / 2}$ by $\exp \left(-\boldsymbol{\iota} \pi\left(1+\boldsymbol{\sigma}_{3}^{1}-\right.\right.$ $\left.\left.\boldsymbol{\sigma}_{3}^{2}\right) / 4\right)$ as in [14], or right-multiply it by $\exp \left(-\boldsymbol{\iota} \pi \boldsymbol{\sigma}_{3}^{1} \mathbf{E}_{-}^{2} / 4\right) \exp \left(\boldsymbol{\iota} \pi \boldsymbol{\sigma}_{3}^{1} \mathbf{E}_{+}^{2} / 4\right)=\exp \left(\boldsymbol{\iota} \pi \boldsymbol{\sigma}_{3}^{1} \boldsymbol{\sigma}_{3}^{2} / 4\right)$ along with $\exp \left(\boldsymbol{\iota} \pi \mathbf{E}_{-}^{2} / 2\right)$ as above. The validation of the Toffoli pulse sequence is similar, though considerably more complex.

An interesting generalization of the two particle conditional rotations is obtained by taking the exponential of all four transitions, i.e.

$$
\begin{aligned}
& \exp \left(-\boldsymbol{\iota}\left(\alpha_{+} \boldsymbol{\sigma}_{1}^{1} \mathbf{E}_{+}^{2}+\alpha_{-} \boldsymbol{\sigma}_{1}^{1} \mathbf{E}_{-}^{2}+\beta_{+} \boldsymbol{\sigma}_{1}^{2} \mathbf{E}_{+}^{1}+\beta_{-} \boldsymbol{\sigma}_{1}^{2} \mathbf{E}_{-}^{1}\right) / 2\right) \\
= & \exp \left(-\boldsymbol{\iota}\left(\left(\alpha_{+}+\alpha_{-}\right) \boldsymbol{\sigma}_{1}^{1} / 4+\left(\beta_{+}-\beta_{-}\right) \boldsymbol{\sigma}_{3}^{1} \boldsymbol{\sigma}_{1}^{2} / 4\right)\right) \\
& \exp \left(-\boldsymbol{\iota}\left(\left(\alpha_{+}-\alpha_{-}\right) \boldsymbol{\sigma}_{1}^{1} \boldsymbol{\sigma}_{3}^{2} / 4+\left(\beta_{+}+\beta_{-}\right) \boldsymbol{\sigma}_{1}^{2} / 4\right)\right) \\
\equiv & \exp (-\iota \mathbf{X}) \exp (-\iota \mathbf{Y})=\exp (-\iota \mathbf{Y}) \exp (-\iota \mathbf{X})
\end{aligned}
$$

where $\mathbf{X}$ and $\mathbf{Y}$ commute. In addition, since

$$
\begin{aligned}
& \mathbf{X}^{2}=\left(\left(\alpha_{+}+\alpha_{-}\right)^{2}+\left(\beta_{+}-\beta_{-}\right)^{2}\right) / 16 \\
& \mathbf{Y}^{2}=\left(\left(\alpha_{+}-\alpha_{-}\right)^{2}+\left(\beta_{+}+\beta_{-}\right)^{2}\right) / 16
\end{aligned}
$$

their propagators can be written in closed form as

$$
\begin{aligned}
& \exp (-\iota \mathbf{X})=\cos \left(\sqrt{\mathbf{X}^{2}}\right)-\iota \sin \left(\sqrt{\mathbf{X}^{2}}\right) \mathbf{X} / \sqrt{\mathbf{X}^{2}} \\
& \exp (-\iota \mathbf{Y})=\cos \left(\sqrt{\mathbf{Y}^{2}}\right)-\iota \sin \left(\sqrt{\mathbf{Y}^{2}}\right) \mathbf{Y} / \sqrt{\mathbf{Y}^{2}}
\end{aligned}
$$

For example, if $\alpha_{+}=\beta_{+}=0$ and $\alpha_{-}=\beta_{-}=\pi \sqrt{2}$, we obtain $\left(\boldsymbol{\sigma}_{3}^{1}+\boldsymbol{\sigma}_{3}^{2}-\boldsymbol{\sigma}_{1}^{1} \boldsymbol{\sigma}_{1}^{2}-\boldsymbol{\sigma}_{2}^{1} \boldsymbol{\sigma}_{2}^{2}\right) / 2$, which is the particle interchange operator $\boldsymbol{\Pi}^{1,2}$ up to a conditional phase of $\exp \left(\boldsymbol{\iota} \pi \mathbf{E}_{+}^{1} \mathbf{E}_{+}^{2}\right)$. If, on the other hand, $\alpha_{+}=\beta_{-}=\pi \sqrt{2}$, we get $\left(\boldsymbol{\sigma}_{3}^{1}-\boldsymbol{\sigma}_{3}^{2}-\boldsymbol{\sigma}_{1}^{1} \boldsymbol{\sigma}_{1}^{2}+\boldsymbol{\sigma}_{2}^{1} \boldsymbol{\sigma}_{2}^{2}\right) / 2$, which is converted by $\exp \left(\boldsymbol{\iota} \pi \mathbf{E}_{+}^{1} \mathbf{E}_{-}^{2}\right)$ into $\frac{1}{2}\left(1-\boldsymbol{\sigma}_{3}^{1} \boldsymbol{\sigma}_{3}^{2}+\boldsymbol{\sigma}_{1}^{1} \boldsymbol{\sigma}_{1}^{2}-\boldsymbol{\sigma}_{2}^{1} \boldsymbol{\sigma}_{2}^{2}\right)$. The terms $1-\boldsymbol{\sigma}_{3}^{1} \boldsymbol{\sigma}_{3}^{2}$ are annihilated by $\mathbf{E}_{ \pm}^{1} \mathbf{E}_{ \pm}^{2}$, while $\boldsymbol{\sigma}_{1}^{1} \boldsymbol{\sigma}_{1}^{2}-\boldsymbol{\sigma}_{2}^{1} \boldsymbol{\sigma}_{2}^{2}$ induces direct transitions between the $\mathbf{E}_{+}^{1} \mathbf{E}_{+}^{2}$ and $\mathbf{E}_{-}^{1} \mathbf{E}_{-}^{2}$ states:

$$
\begin{aligned}
& \left(\boldsymbol{\sigma}_{1}^{1} \boldsymbol{\sigma}_{1}^{2}-\boldsymbol{\sigma}_{2}^{1} \boldsymbol{\sigma}_{2}^{2}\right) \mathbf{E}_{ \pm}^{1} \mathbf{E}_{ \pm}^{2}\left(\boldsymbol{\sigma}_{1}^{1} \boldsymbol{\sigma}_{1}^{2}-\boldsymbol{\sigma}_{2}^{1} \boldsymbol{\sigma}_{2}^{2}\right) \\
= & \mathbf{E}_{\mp}^{1} \mathbf{E}_{\mp}^{2}\left(\boldsymbol{\sigma}_{1}^{1} \boldsymbol{\sigma}_{1}^{2}-\boldsymbol{\sigma}_{2}^{1} \boldsymbol{\sigma}_{2}^{2}\right)^{2}=\mathbf{E}_{\mp}^{1} \mathbf{E}_{\mp}^{2}\left(2+2 \boldsymbol{\sigma}_{3}^{1} \boldsymbol{\sigma}_{3}^{2}\right)=4 \mathbf{E}_{\mp}^{1} \mathbf{E}_{\mp}^{2} .
\end{aligned}
$$

We note that these transformations can be implemented in NMR via what might be called compound pulses, i.e. multiple simultaneous pulses each selective for a single transition [15,22].

Conditional rotations further enable us to diagonalize a general two-spin NMR Hamiltonian $\mathbf{H}^{1,2}$ as above. If we define the correlated idempotents $\mathbf{E}_{ \pm}^{1,2} \equiv \frac{1}{2}\left(1 \pm \boldsymbol{\sigma}_{3}^{1} \boldsymbol{\sigma}_{3}^{2}\right)$, this may be rewritten as 


$$
\mathbf{H}^{1,2}=\frac{1}{2} \omega^{1} \boldsymbol{\sigma}_{3}^{1}+\frac{1}{2} \omega^{2} \boldsymbol{\sigma}_{3}^{2}+\pi J^{1,2}\left(\frac{1}{2} \boldsymbol{\sigma}_{3}^{1} \boldsymbol{\sigma}_{3}^{2}+\boldsymbol{\sigma}_{1}^{1} \boldsymbol{\sigma}_{1}^{2} \mathbf{E}_{-}^{1,2}\right)
$$

We can split $\mathbf{H}^{1,2}$ into two commuting parts as follows:

$$
\mathbf{H}^{1,2}=\mathbf{H}^{1,2} \mathbf{E}_{+}^{1,2}+\mathbf{H}^{1,2} \mathbf{E}_{-}^{1,2} \equiv \mathbf{H}_{+}^{1,2}+\mathbf{H}_{-}^{1,2}
$$

Since $\mathbf{E}_{+}^{1,2} \mathbf{E}_{-}^{1,2}=0$, the $\mathbf{H}_{+}^{1,2}$ part is already diagonal. The off-diagonal part, on the other hand, can be written as

$$
\mathbf{H}_{-}^{1,2}-\frac{1}{2} \pi J^{1,2} \mathbf{E}_{-}^{1,2}=\left(\omega_{-} \boldsymbol{\sigma}_{3}^{1}+\pi J^{1,2} \boldsymbol{\sigma}_{1}^{1} \boldsymbol{\sigma}_{1}^{2}\right) \mathbf{E}_{-}^{1,2}
$$

where $\omega_{ \pm} \equiv \omega^{1} \pm \omega^{2}$. The $\boldsymbol{\sigma}_{3}^{1}$ and $\boldsymbol{\sigma}_{1}^{1} \boldsymbol{\sigma}_{1}^{2}$ terms are rotated into one another by $\exp \left(\phi \boldsymbol{\iota} \boldsymbol{\sigma}_{2}^{1} \boldsymbol{\sigma}_{1}^{2}\right)$, and hence this can be written in polar form as

$$
\mathbf{H}_{-}^{1,2}-\frac{1}{2} \pi J^{1,2} \mathbf{E}_{-}^{1,2}=\Theta \boldsymbol{\sigma}_{3}^{1} \exp \left(\phi \iota \boldsymbol{\sigma}_{1}^{1} \boldsymbol{\sigma}_{1}^{2}\right) \mathbf{E}_{-}^{1,2}
$$

where $\Theta \equiv \sqrt{\left(\omega_{-}\right)^{2}+\left(\pi J^{1,2}\right)^{2}}$ and $\phi \equiv \arctan \left(\pi J^{1,2} / \omega_{-}\right)$. The right-hand side of this equation is clearly diagonalized by $\exp \left(-\phi \boldsymbol{\iota} \boldsymbol{\sigma}_{2}^{1} \boldsymbol{\sigma}_{1}^{2} / 2\right)$ and commutes with $\mathbf{E}_{-}^{1,2}$, from which it follows that $\mathbf{H}^{1,2}$ is diagonalized by the conditional rotation

$$
\mathbf{T}(\phi) \equiv e^{-\phi \boldsymbol{\iota} \sigma_{2}^{1} \boldsymbol{\sigma}_{1}^{2} \mathbf{E}_{-}^{1,2} / 2}=e^{-\phi \boldsymbol{\iota} \boldsymbol{\sigma}_{1}^{1} \boldsymbol{\sigma}_{1}^{2} / 2} \mathbf{E}_{-}^{1,2}+\mathbf{E}_{+}^{1,2}
$$

The result is a weak coupling Hamiltonian whose frequencies have been shifted by $\pm \Theta$,

$$
\tilde{\mathbf{T}}(\phi) \mathbf{H}^{1,2} \mathbf{T}(\phi)=\frac{1}{2}\left(\omega_{+}+\Theta\right) \boldsymbol{\sigma}_{3}^{1}+\frac{1}{2}\left(\omega_{+}-\Theta\right) \boldsymbol{\sigma}_{3}^{2}+\frac{1}{2} \pi J^{1,2} \boldsymbol{\sigma}_{3}^{1} \boldsymbol{\sigma}_{3}^{2},
$$

while the corresponding transition moment shows that the peak intensities have been altered by

$$
\begin{aligned}
& \left\langle\left[\mathbf{E}_{\epsilon_{1}}^{1} \tilde{\mathbf{T}}(\phi)\left(\boldsymbol{\sigma}_{1}^{1}+\boldsymbol{\sigma}_{1}^{2}\right) \mathbf{T}(\phi) \mathbf{E}_{\epsilon_{1}^{\prime}}^{1}\right]\left[\mathbf{E}_{\epsilon_{2}}^{2} \tilde{\mathbf{T}}(\phi)\left(\boldsymbol{\sigma}_{1}^{1}+\boldsymbol{\sigma}_{1}^{2}\right) \mathbf{T}(\phi) \mathbf{E}_{\epsilon_{2}^{\prime}}^{2}\right]\right\rangle \\
= & \left(1+\epsilon_{1} \sin (\phi)\right) \delta_{\epsilon_{1}, \epsilon_{1}^{\prime}} \delta_{\epsilon_{2},-\epsilon_{2}^{\prime}}+\left(1-\epsilon_{2} \sin (\phi)\right) \delta_{\epsilon_{1},-\epsilon_{1}^{\prime}} \delta_{\epsilon_{2}, \epsilon_{2}^{\prime}} .
\end{aligned}
$$

The Hadamard transform plays an essential role in many quantum algorithms, but its simple geometric interpretation is seldom pointed out. Consider a rotation of the $m$-th particle by an angle $\alpha$ about the $\mathbf{w}^{m} \equiv\left(\boldsymbol{\sigma}_{1}^{m}+\boldsymbol{\sigma}_{3}^{m}\right) / \sqrt{2}$ axis:

$$
\mathbf{W}^{m}(\alpha) \equiv \exp \left(-\iota \alpha \mathbf{w}^{m} / 2\right)=\cos (\alpha / 2)-\iota \mathbf{w}^{m} \sin (\alpha / 2)
$$

The one-particle Hadamard transform $\mathbf{W}^{m}=-\iota \mathbf{w}^{m}$ is obtained when $\alpha=\pi$, and the $N$-particle Hadamard transform $\mathbf{W}_{N}$ is simply the commutative product of the Hadamard transforms of the individual particles. The quantum Fourier transform (QFT) [23] can likewise be written, and simplified, using the multiparticle geometric algebra. As shown by Don Coppersmith (unpublished manuscript), the $N$-particle QFT $\mathbf{Q}_{N}$ can be written as a recursive product of one-particle Hadamard transforms and two-particle conditional phase shifts,

$$
\mathbf{V}^{\ell, m} \equiv \exp \left(-\iota \omega_{\ell m} \mathbf{E}_{+}^{\ell} \mathbf{E}_{+}^{m}\right) \quad\left(\omega_{\ell m} \equiv \pi 2^{\ell-m} \text { for } \ell \leq m\right)
$$


namely

$$
\mathbf{Q}_{N} \equiv \mathbf{U}^{1} \cdots \mathbf{U}^{N-1} \mathbf{U}^{N} \equiv\left(\mathbf{W}^{1} \mathbf{V}^{1,2} \cdots \mathbf{V}^{1, N}\right) \cdots\left(\mathbf{W}^{N-1} \mathbf{V}^{N-1, N}\right)\left(\mathbf{W}^{N}\right) .
$$

Each factor $\mathbf{U}^{m}$ above can be rearranged so that all the Hadamaard transformations come first:

$$
\begin{aligned}
\mathbf{U}^{m} & =\left(-\iota \mathbf{w}^{m}\right)\left(e^{-\imath \omega_{m, m+1} \mathbf{E}_{+}^{m} \mathbf{E}_{+}^{m+1}} \cdots e^{-\iota \omega_{m, N} \mathbf{E}_{+}^{m} \mathbf{E}_{+}^{N}}\right)\left(\iota \mathbf{w}^{m}\right)\left(-\iota \mathbf{w}^{m}\right) \\
& =e^{-\iota\left(-\iota \mathbf{w}^{m}\right) \mathbf{E}_{+}^{m}\left(\iota \mathbf{w}^{m}\right)\left(\omega_{m, m+1} \mathbf{E}_{+}^{m+1}+\cdots+\omega_{m, N} \mathbf{E}_{+}^{N}\right)}\left(-\iota \mathbf{w}^{m}\right) \\
& =e^{-\iota / 2} e^{-\iota \boldsymbol{\sigma}_{1}^{m}\left(\omega_{m, m+1} \mathbf{E}_{+}^{m+1}+\cdots+\omega_{m, N} \mathbf{E}_{+}^{N}\right)}\left(-\iota \mathbf{w}^{m}\right)
\end{aligned}
$$

Since the factors $\mathbf{U}^{n}$ with $n>m$ do not involve the $m$-th particle space, we may therefore write the QFT with all the $-\iota \mathbf{w}^{m}$ together on one side, i.e.

$$
\mathbf{Q}_{N}=e^{-N \iota / 2} e^{-\iota \boldsymbol{\sigma}_{1}^{1}\left(\omega_{1,2} \mathbf{E}_{+}^{2}+\cdots+\omega_{1, N} \mathbf{E}_{+}^{N}\right)} \cdots e^{-\iota \boldsymbol{\sigma}_{1}^{N-1}\left(\omega_{N-1, N} \mathbf{E}_{+}^{N}\right)} \mathbf{W}_{N} .
$$

In NMR spectroscopy, each of the conditional rotations in this expression can in principle be implemented with a single compound pulse. The Hadamard transform $\mathbf{W}_{N}$ of all $N$ spins, on the other hand, can be implemented with three "hard" (nonselective) pulses, namely $\exp \left(\boldsymbol{\iota} \pi \boldsymbol{\sigma}_{2} / 8\right) \exp \left(\boldsymbol{\iota} \pi \boldsymbol{\sigma}_{1} / 2\right) \exp \left(-\boldsymbol{\iota} \pi \boldsymbol{\sigma}_{2} / 8\right)$, which takes only a very small fraction of the time required for a "soft" pulse (or the conditional rotations). The complexity of this NMR implementation of the QFT is only $O(N)$, and since the time required for a hard pulse is essentially independent of the number of spins involved, the implementation requires only half the number of steps in the parallel implementation proposed by Coppersmith.

In closing, we note one final advantage of multiparticle geometric algebra, which is the astonishing ease with which one can compute partial traces. In terms of idempotents, the partial trace of a density matrix $\boldsymbol{\rho}$ over any one particle $m$ can be written as:

$$
\operatorname{Tr}_{m}(\boldsymbol{\rho})=\mathbf{E}_{+}^{m} \boldsymbol{\rho} \mathbf{E}_{+}^{m}+\mathbf{E}_{-}^{m} \boldsymbol{\rho} \mathbf{E}_{-}^{m}+\boldsymbol{\sigma}_{1}^{m}\left(\mathbf{E}_{+}^{m} \boldsymbol{\rho} \mathbf{E}_{+}^{m}+\mathbf{E}_{-}^{m} \boldsymbol{\rho} \mathbf{E}_{-}^{m}\right) \boldsymbol{\sigma}_{1}^{m}
$$

Consider, for example, the three particle GHZ state, $\boldsymbol{\rho}_{\mathrm{GHZ}} \equiv$

$$
\left(1+\boldsymbol{\sigma}_{3}^{1} \boldsymbol{\sigma}_{3}^{2}+\boldsymbol{\sigma}_{3}^{1} \boldsymbol{\sigma}_{3}^{3}+\boldsymbol{\sigma}_{3}^{2} \boldsymbol{\sigma}_{3}^{3}+\boldsymbol{\sigma}_{1}^{1} \boldsymbol{\sigma}_{1}^{2} \boldsymbol{\sigma}_{1}^{3}-\boldsymbol{\sigma}_{2}^{1} \boldsymbol{\sigma}_{2}^{2} \boldsymbol{\sigma}_{1}^{3}-\boldsymbol{\sigma}_{2}^{1} \boldsymbol{\sigma}_{1}^{2} \boldsymbol{\sigma}_{2}^{3}-\boldsymbol{\sigma}_{1}^{1} \boldsymbol{\sigma}_{2}^{2} \boldsymbol{\sigma}_{2}^{3}\right) / 8 .
$$

Using the relations $\mathbf{E}_{ \pm}^{3} \boldsymbol{\sigma}_{k}^{3} \mathbf{E}_{ \pm}^{3}=\mathbf{E}_{ \pm}^{3} \mathbf{E}_{\mp}^{3} \boldsymbol{\sigma}_{k}^{3}=0$ and $\boldsymbol{\sigma}_{k}^{3} \mathbf{E}_{ \pm}^{3} \boldsymbol{\sigma}_{k}^{3}=\mathbf{E}_{\mp}^{3}$ for $k=1,2$, one finds that

$$
\mathbf{E}_{ \pm}^{3} \boldsymbol{\rho}_{\mathrm{GHZ}} \mathbf{E}_{ \pm}^{3}=\mathbf{E}_{ \pm}^{3}\left(1+\boldsymbol{\sigma}_{3}^{1} \boldsymbol{\sigma}_{3}^{2}+\boldsymbol{\sigma}_{3}^{1} \boldsymbol{\sigma}_{3}^{3}+\boldsymbol{\sigma}_{3}^{2} \boldsymbol{\sigma}_{3}^{3}\right) / 8
$$

and

$$
\boldsymbol{\sigma}_{1}^{3} \mathbf{E}_{ \pm}^{3} \boldsymbol{\rho}_{\mathrm{GHZ}} \mathbf{E}_{ \pm}^{3} \boldsymbol{\sigma}_{1}^{3}=\mathbf{E}_{\mp}^{3}\left(1+\boldsymbol{\sigma}_{3}^{1} \boldsymbol{\sigma}_{3}^{2}-\boldsymbol{\sigma}_{3}^{1} \boldsymbol{\sigma}_{3}^{3}-\boldsymbol{\sigma}_{3}^{2} \boldsymbol{\sigma}_{3}^{3}\right) / 8
$$

The sum of the four terms in Eq. (40) is thus the mixed state

$$
\operatorname{Tr}_{3}\left(\boldsymbol{\rho}_{\mathrm{GHZ}}\right)=\left(1+\boldsymbol{\sigma}_{3}^{1} \boldsymbol{\sigma}_{3}^{2}\right) / 4 \text {. }
$$

More generally, the partial trace is obtained simply by dropping all terms from the density matrix containing factors from the particles over which the trace is taken, and multiplying by two to the number of such particles. 
In conclusion, we have shown how to formulate the most important operations of quantum computing in multiparticle geometric algebra, and illustrated some of the advantages that this more general theory has over the established product operator formalism used in NMR spectroscopy. These advantages will be further demonstrated in subsequent publications devoted to developing the theory and methods needed for ensemble quantum computing by NMR spectroscopy.

\section{Acknowledgements}

This work was supported by, or in part by, the U. S. Army Research Office under contract/grant number DAAG 55-97-1-0342 from the DARPA Ultrascale Computing Program. 


\section{REFERENCES}

[1] D. Hestenes and G. Sobczyk. Clifford Algebra to Geometric Calculus. D. Reidel Pub. Co., Dordrecht, NL, 1984.

[2] D. Hestenes. New Foundations for Classical Mechanics. D. Reidel Pub. Co., Dordrecht, NL, 1986.

[3] W. E. Baylis. Clifford (Geometric) Algebras, with Applications in Physics, Mathematics, and Engineering. Birkhauser, Boston, MA, 1996.

[4] P. Lounesto. Clifford Algebras and Spinors. London Math. Soc. Lect. Notes, \#239. Cambridge Univ. Press, Cambridge, U.K., 1997.

[5] T. F. Havel and I. Najfeld. J. Mol. Struct. (TheoChem), 308:241-262, 1994.

[6] T. F. Havel and I. Najfeld. J. Mol. Struct. (TheoChem), 336:175-189, 1995.

[7] C. J. L. Doran, A. N. Lasenby, and S. F. Gull. Found. Phys., 23:1239-1264, 1993.

[8] C. J. L. Doran, A. N. Lasenby, S. F. Gull, S. S. Somaroo, and A. D. Challinor. Spacetime algebra and electron physics. In P. Hawkes, editor, Advances in Imaging and Electron Physics, pages 271-386. Academic Press, Englewood Cliffs, NJ, 1996.

[9] S. Lloyd. Science, 263:1569-1571, 1993.

[10] G. Brassard. A quantum jump in computer science. In J. van Leeuwen, editor, Computer Science Today, volume 1000 of Lect. Notes Comput. Sci., pages 1-14. Springer-Verlag, 1995.

[11] D. Divincenzo. Science, 270:255-261, 1995.

[12] N. A. Gershenfeld and I. L. Chuang. Science, 275:350-356, 1997.

[13] D. G. Cory, A. F. Fahmy, and T. F. Havel. Proc. Natl. Acad. Sci., 94:1634-1639, 1997.

[14] D. G. Cory, M. D. Price, and T. F. Havel. Nuclear magnetic resonance spectroscopy: An experimentally accessible paradigm for quantum computing. Physica D, 1997. In press (available as LANL preprint quant-ph/9709001).

[15] O. W. Sörensen, G. W. Eich, M. H. Levitt, G. Bodenhausen, and R. R. Ernst. Product operator formalism for the description of NMR pulse experiments. Prog. NMR Spect., 16:163-192, 1983.

[16] F. J. M. van de Ven and C. W. Hilbers. J. Magn. Reson., 54:512-520, 1983.

[17] R. R. Ernst, G. Bodenhausen, and A. Wokaun. Principles of Nuclear Magnetic Resonance in One and Two Dimensions. Oxford Univ. Press, U.K., 1987.

[18] B. Boulat and M. Rance. Mol. Phys., 83:1021-1039, 1994.

[19] B. Blümich and H. W. Spiess. J. Maget. Reson., 61:356-362, 1985.

[20] C. Counsell, M. H. Levitt, and R. R. Ernst. J. Magn. Reson., 63:133-141, 1985.

[21] D. J. Siminovitch. Part I. Concepts Magn. Reson., 9:149-171, 1997; Part II. Concepts Magn. Reson., 9:211-225, 1997.

[22] H. Hatanaka and C. S. Yannoni. J. Magn. Reson., 42:330-333, 1981.

[23] P. W. Shor. SIAM J. Comput., 26:1484-1509, 1997. 